BRITISH MEDICAL JOURNAL VOLUME $286 \quad 16$ APRIL 1983

' Graham WR. Adverse effects of dapsone. Int $\mathcal{F}$ Dermatol 1975;14:494-500.

2 Ognibene AJ. Agranulocytosis due to dapsone. Ann Intern Med 1970;72: 521-4.

${ }^{3}$ Ognibene AJ. Why agranulocytosis from dapsone? Ann Intern Med 1972; 77:153.

4 Report of Meetings Convened by the Ross Institute. Malaria prevention in travellers from the United Kingdom. Br Med f $1981 ; 283: 214-8$.

${ }^{5}$ Weetman RM, Boxer LA, Brown MP, Mantich NM, Baehner RL. In vitro inhibition of granulopoiesis by 4-amino-4'-hydroxylaminodiphenyl sulfone. Br f Haematol 1980;45:361-70.

(Accepted 20 fanuary 1983)

Department of Infectious Diseases, Uppsala University Hospital, Uppsala, Sweden

GÖRAN FRIMAN, MD

CHRISTINA NYSTRÖM-ROSANDER, MD

GUDRUN JONSELL, MD

Department of Infectious Diseases, Karolinska Institute, Stockholm, Sweden

ANDERS BJÖRKMAN, MD

Department of Infectious Diseases, County Hospital, Gävle, Sweden GÖRAN LEKÅS, MD

Department of Infectious Diseases, County Hospital, Jönköping, Sweden

BERIT SVENDSRUP, MD

Correspondence to: Christina Nyström-Rosander, MD, Department of Infectious Diseases, University Hospital, S-751 85 Uppsala, Sweden.

\section{Unusual case of peripheral venous occlusion}

We describe a case in which the use of a proprietary sexual aid necessitated surgical intervention to effect its removal. Investigation showed these items to be in common usage.

\section{Case history}

A 21 year old man presented to the accident and emergency department on the morning of 24 December 1981. For three years he had been in the habit of using a so called "cock ring." The device consisted of a simple metal ring which was fitted around the base of the penile shaft proximal to the scrotum before erection. Erection would cause the ring to tighten, thus occluding venous return and resulting in maintenance and prolongation of the turgid state of the penis.

Having recently misiaid his usual ring, the patient had purchased a new one, which he had used for the first time the previous hight, some 12 hours before presentation. Although enjoying satisfactory intercourse, he had been unable to remove the ring either immediately after the act or on waking the next morning. The ring was causing pain and he was unable to urinate.

Examination showed the penis to be congested and semierect (figure). There was moderate scrotal oedema and the ring was firmly in situ around the base of the penile shaft, obstructing venous return. All attempts to pull the ring off were unsuccessful. A rotary cutter used to remove rings after finger injuries made no progress and finally broke. Eventually the ring was cut in two places with a hacksaw, the patient sustaining a small scrotal laceration in the process. The ring (figure) was $5 \mathrm{~cm}$ internal diameter and the metal $0.8 \mathrm{~cm}$ in cross section.

\section{Comment}

Similar rings are apparently readily found in shops supplying sexual aids in and around central London. They are available in various sizes and no warning is supplied. The possibility of the reported complication of the use of these rings is inherent in their mode of action In more extreme cases they could conceivably cause penile ischaemia or even necrosis.

We think that rings of this type should be withdrawn from circulation, and since there seems to be a demand they should be replaced by models with a clasp and hinge mechanism for rapid release.

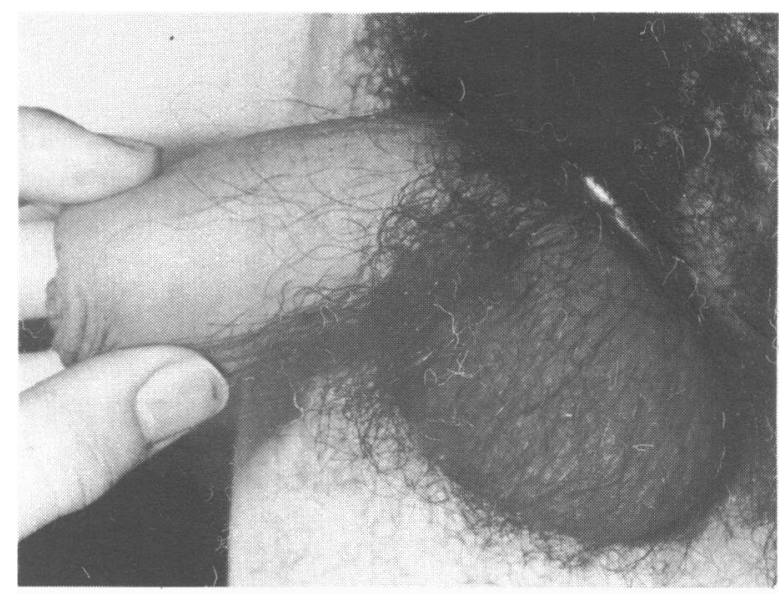

Above: Appearance at presentation. Below: The ring after removal.

Alternatively they might be made of rubber or a similar elastic material, which could be stretched if necessary.

(Accepted 12 fanuary 1983)

Accident and Emergency Department, Middlesex Hospital, London W1

D G BOGOD, $\mathrm{MB}$, BS, senior house officer

A J POPE, $M B$, BS, senior house officer

Correspondence to: Dr D G Bogod, Department of Anaesthetics, Leiceste Royal Infirmary, Leicester LE1 5WW.

\section{Identical twins with identical vesicoureteric reflux: chronic pyelonephritis in one}

It is well established that chronic pyelonephritic scarring occurs predominantly in kidneys drained by ureters in which vesicoureteric reflux occurs in childhood. ${ }^{1}$ Most of such ureters develop a competent vesicoureteric valve during follow up periods of at least 10 years, ${ }^{2}$ so that only a few adults with pyelonephritic scarring still show reflux. ${ }^{3}$ Reflux of sufficient severity to be associated with renal scarring is inherited as an autosomal dominant with high penetrance. ${ }^{4}$ Controversy continues as to whether reflux per se causes renal scarring during a period of high vulnerability in early childhood or whether the kidney is only damaged when the refluxing urine is infected. ${ }^{3}$ We describe identical twins in whom bilateral vesicoureteric reflux persisted into adult life, only one of whom developed chronic pyelonephritis, suggesting that an environmental factor is required before reflux leads to scarring. 

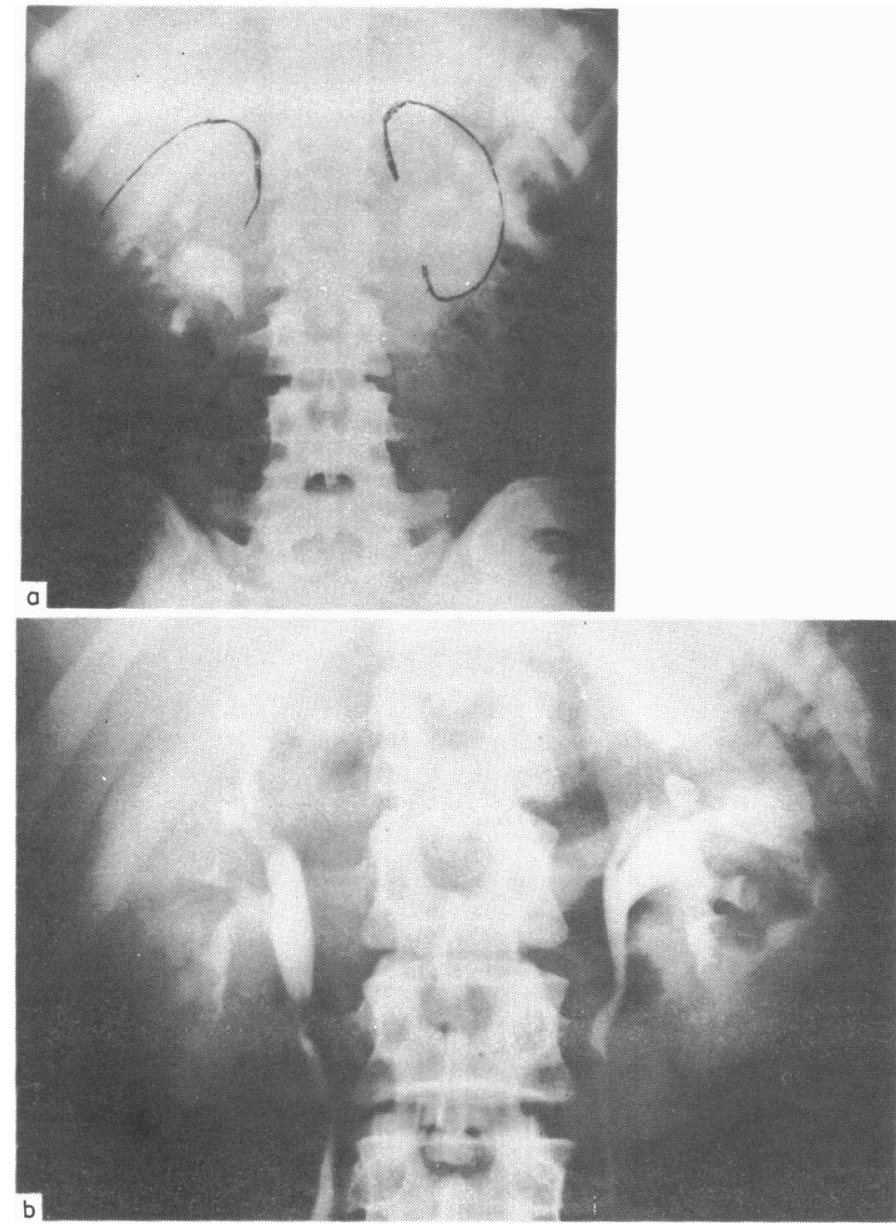

Pyelograms from two identical twins. (a) Case 1, showing left chronic pyelonephritis; renal outlines have been pencilled in for clarity. (b) Case 2, showing normal appearances apart from a minor degree of duplex bilaterally.

\section{Case reports}

Case 1-This patient was investigated at the age of 18 for recurrent urinary infection. She had been aware of symptoms only for the preceding few months. The following year intravenous urography showed a normal right kidney and a shrunken left kidney with clubbing of the calyces (fig (a)) consistent with chronic pyelonephritis. On micturating cystography there was reflux up the left ureter as far as the renal pelvis but without dilatation of the renal tract. Cystoscopy showed a localised trigonitis. She was treated initially by urethral dilatation but, after a further recurrence of infection with haematuria, she underwent left nephrectomy at the age of 19 . Histological examination confirmed the presence of severe chronic pyelonephritis. She continued to suffer from urinary infection and haematuria in the year after nephrectomy, at which point she was lost to follow up.

Case 2 is the twin sister of case 1 . They were always regarded as identical, and identity was confirmed in 19 red cell types and the four HLA-A and HLA-B types (courtesy of Dr Ann Collins). She presented to one of us in general practice with a history of recurrent urinary infection, in which the first proved attack was at the age of 24 . In view of the family history she was referred for investigation; no evidence of chronic pyelonephritis was seen on intravenous urogram (fig $(b)$ ). Micturating cystogram showed reflux up the left ureter of similar extent to that shown in her twin sister and also slight reflux up the right ureter. She was followed up as an outpatient for about three years, during which time she ceased to suffer from urinary infections. Repeat intravenous urography after five years confirmed the normality of the kidneys. Analysis of urine gave consistently normal results, and plasma urea and creatinine concentrations were within normal ranges.

\section{Comment}

The chronic pyelonephritis in case 1 was obvious on the intravenous urogram and its widespread presence in the kidney was confirmed by histological examination. Her twin sister's left kidney was not, of course, examined histologically but it appeared entirely normal on two intravenous urograms giving a good demonstration of the calyceal system and on the nephrogram. The likeliest explanation for the different behaviour of the kidneys in two identical twins is that one contracted a urinary infection in infancy, when the kidneys were susceptible to reflux of infected urine, and the other did not. We cannot prove this hypothesis because our patient, like most of those in whom chronic pyelonephritis is diagnosed in adult life, had no recollection of infection in early childhood; at that age the symptoms are non-specific and they are often forgotten by the time the diagnosis is made 20 years later.

1 Scott JES. The management of ureteric reflux in children. $\mathrm{Br} \mathcal{F}$ Urol 1977;49:109-18.

2 Smellie J, Edwards D, Hunter N, Normand ICS, Prescod N. Vesicoureteric reflux and renal scarring. Kidney Int 1975;4,suppl:65-72.

${ }^{3}$ Arze RS, Ramos JM, Owen JP, et al. The natural history of chronic pyelonephritis in the adult. $Q \mathcal{F}$ Med 1982;51:396-410.

4 Parsons V, Munson KW, Thompson P, Packham D, Kennedy L. Renal reflux, scarring, cystometrography and tissue types. Proc Eur Dial Transplant Assoc 1979;16:542-50.

(Accepted 25 fanuary 1983)

University Department of Medicine, Royal Victoria Infirmary, Newcastle upon Tyne NE1 4LP

D N S KERR, MSC, FRCP, professor of medicine

Jarrow, Tyne and Wear

P M PILLAI, MB, DCH, general practitioner

Correspondence to: Dr D N S Kerr.

\section{Clobazam as adjunctive treatment in refractory epilepsy}

The benzodiazepines have an established role in the treatment of epileptic seizures. Diazepam finds use either intravenously or per rectum in the management of status epilepticus, and several of the 1,4 structure have been evaluated for oral use in long term management of intractable seizures. More recently 1,5-benzodiazepines have been introduced, which are claimed to have fewer side effects but retain their therapeutic anticonvulsant potential. ${ }^{1}$ Clobazam has recently been evaluated as an anticonvulsant, and preliminary trials indicate its potential value as adjunctive treatment in epilepsy. ${ }^{2-4}$

We present the results of a double blind trial of clobazam versus placebo in patients with chronic poorly controlled epilepsy.

\section{Patients, methods, and results}

We studied 26 patients with a mean age of 34 (range 18-60) years. All were resident at the Chalfont Centre for Epilepsy and were having four or more uncontrolled seizures a month. Patients were given $30 \mathrm{mg}$ clobazam nightly or an identically matched placebo capsule in a double blind cross over design. Half received the active treatment first, and each medication period was nine weeks. Between treatments there was an eight week washout period, and after treatment tablets were withdrawn over one week. Patients were seen at weekly intervals for assessment of side effects and dose adjustment. Early morning fasting blood samples were taken in each of the last three weeks of treatment for measurement of serum concentrations of clobazam, desmethylclobazam, and concomitant antiepileptic drugs.

Six patients withdrew from the study, one during the placebo period. The table shows the frequency of seizures during each treatment. There was a significant fall in frequency during the active treatment period ( $p=$ 0.002 ), especially of partial seizures. Three patients had no seizures and 12 had a $50 \%$ or more reduction with clobazam. When patients who withdrew were included as non-responders the number of patients showing a $50 \%$ or greater fall in seizures remained significant $\left(\chi^{2}=14.3 ; \mathrm{p}<0.01\right)$. Period or interaction effects were not seen.

Significantly more seizures were seen in the clobazam withdrawal period $(p<0.02)$, but no significant tolerance to the drug effect was noted in those patients showing a response. (Tolerance was assessed by noting the frequency of seizures in the first and last halves of the active treatment period.)

Adverse effects occurred more often during the clobazam period: of the 20 patients staying in the trial, treatment was reduced because of side effects in six taking clobazam and two taking placebo. Mood changes recorded included irritability, depression, and disinhibition.

No significant changes in concentrations of other anticonvulsants were seen (table), although only a few patients were taking phenobarbitone, phenytoin, and sodium valproate. Serum concentrations of clobazam and desmethylclobazam were not significantly related to the therapeutic response.

(1)

要

